\title{
Device Incorrectly Assembled During Reprocessing
}

National Cancer Institute

\section{Source}

National Cancer Institute. Device Incorrectly Assembled During Reprocessing. NCI

Thesaurus. Code C139485.

Incorrect assembly of the device following reprocessing. 